\title{
Karyological Study of Tusker and Tuskless Male Asian Elephant (Elephas maximus) by Conventional, GTG-, and Ag-NOR Banding Techniques
}

\author{
Sukjai Rattanayuvakorn ${ }^{1}$, Alongklod Tanomtong ${ }^{2 *}$, Sumalee Phimphan ${ }^{2}$, \\ Wiwat Sangpakdee ${ }^{3}$, Sitthisak Pinmongkhonkul ${ }^{4}$ and Krit Phintong ${ }^{5}$ \\ ${ }^{1}$ Department of Science and Mathematics, Faculty of Agriculture and Technology, Rajamangala University of \\ Technology Isan, Surin Campus, Muang, Surin 32000, Thailand \\ ${ }^{2}$ Toxic Substances in Livestock and Aquatic Animals Research Group, Department of Biology, Faculty of Science, \\ Khon Kaen University, Muang, Khon Kaen 40002, Thailand \\ ${ }^{3}$ Biology program, Faculty of Science, Udon Thani Rajabhat University, Muang, Udon Thani 40001, Thailand \\ ${ }^{4}$ Department of Biology, School of Science, University of Phayao, Tumbol Maeka, Muang, Phayao 56000, \\ Thailand \\ ${ }^{5}$ Department of Fundamental Science, Faculty of Science and Technology, Surindra Rajabhat University, Muang, \\ Surin 32000, Thailand
}

Received April 19, 2016; accepted June 3, 2017

\begin{abstract}
Summary The present study was undertaken to identify whether any difference exists in karyotype between the tusker (chang Phai) and tuskless (chang Sridor) male Asian elephants (Elephas maximus, Linn. 1758). The elephant using in the present work are owned of some local people who lived in the elephant village, Surin Province, Thailand. Peripheral bloods were collected from the ear-vein of healthy elephants. The lymphocyte culture and harvesting method was employed follow standard protocols. Conventional, GTG-, and Ag-NORs banding techniques were applied to find out the different of both elephant karyotypes. The results showed that the standardized karyotype of both elephants were consisted of one pair of large acrocentric, four pairs of large telocentric, one pair of medium submetacentric, one pair of medium acrocentric, six pairs of medium telocentric, two pairs of small metacentric, two pairs of small submetacentric, and ten pairs of small telocentric homologous chromosomes. The X sex-chromosome was a medium metacentric and the Y sex-chromosome was a small acrocentric chromosome. Here, the NORs bearing chromosome were found on one pair of small submetacentric and two pairs of small telocentric chromosomes similar in both of male elephants. However, those classical cytogenetic techniques could not be distinguished the karyotype of tusker and tuskless male elephants. At least, we present the standardized karyotype, idiogram and the basic cytogenetic data that could be served as a basis for future chromosome analyses of Asian elephant populations and for comparative cytogenetic studies with other related species. The karyotype formula could be deduced as:
\end{abstract}

$2 n$ (diploid) $=\mathrm{L}_{2}^{\mathrm{a}}+\mathrm{L}_{8}^{\mathrm{t}}+\mathrm{M}_{2}^{\mathrm{sm}}+\mathrm{M}_{2}^{\mathrm{a}}+\mathrm{M}_{12}^{\mathrm{t}}+\mathrm{S}_{4}^{\mathrm{m}}+\mathrm{S}_{4}^{\mathrm{sm}}+\mathrm{S}_{20}^{\mathrm{t}}+\mathrm{XY}$.

Key words Karyotype, Elephas maximus, Asian elephant, Tusker elephant, Tuskless elephant.

The elephant is classified under the order Proboscidea, family Elephantudae, subfamilies Stegotetra, Belontidae, and Elephantinae, with a total of six genera and 26 species. However, there are only two genera existing in this world today. The Asian or Indian elephant (Elephas maximus) and African elephant (Loxodonta africana) are represented of the family Elephantidae. Elephants believed to have originated in Africa and then radiated to Asia, Europe, and North America. Elephants are the largest land mammals, with male E. maximus stand around $300 \mathrm{~cm}$ at the shoulder and weigh $5400 \mathrm{~kg}$ (Houck et al. 2001). Basically, elephants have specialized dentition, including tusks, which are elongated

\footnotetext{
* Corresponding author, e-mail: tanomtong@hotmail.com DOI: $10.1508 /$ cytologia.82.349
}

second upper incisors and are more prominent in males while female E. maximus often lack tusks. Elephant are listed as endangered, and a continued decline of elephant populations is expected so far (Vaughn 1986, Nowak 1999).

Previously, classical cytogenetic studies have been carried out for both the L. africana (Hungerford et al. 1966, Hösli and Thürig 1970, Thürig 1970, Hsu and Benirschke 1971a, Wallace 1978) and E. maximus (Hungerford et al. 1966, Norberg 1969, Hsu and Benirschke 1971b, Sakthikumar et al. 1990, Hartl et al. 1995, Suwattana et al. 2000, Graphodatsky et al. 2002, Sharudin 2005). Houck et al. (2001) reported comparative cytogenetics of L. africana and E. maximus, then up to date the comparative chromosome painting based on fluorescence in situ hybridization (FISH) have been studied on 
both elephant species. Comparisons between the species demonstrated a high level of chromosome band homology, with 26 conserved autosomal pairs.

In Thailand, there are two types of male elephants, the first one called chang Phai (tusker) possess prominent tusks which are outgrowths of the second upper incisors. Another type of male elephant (tuskless) known as chang Sridor possesses a rudimentary outgrowth of the incisors or absent (Fig. 1). The present study was undertaken to identify whether there exits any difference in chromosome morphology, GTG-dark/light band and NORs location on between the tusker and tuskless male elephants.

\section{Materials and methods}

The elephant using in the present work are owned of some local people who lived in the elephant village, Surin province, Thailand. Peripheral bloods were collected from the ear-vein of healthy tusker and tuskless male elephants. The lymphocyte culture method using whole blood was employed (Rooney 2001) with some modification, $0.8 \mathrm{~mL}$ of whole blood were added to the culture media which were performed using a mixture of $80 \%$ RPMI $1640,20 \%$ fetal bovine serum, $0.1 \mathrm{~mL}$ of pokeweed mitogen $\left(40 \mu \mathrm{g} \mathrm{mL}^{-1}\right)$ and $0.1 \mathrm{~mL}$ of pen-strep antibiotic. The culture were incubated at $37^{\circ} \mathrm{C}$ for $72 \mathrm{~h}$ and mixed gently twice daily, one drop of $0.01 \%$ colchicine were added at $71 \mathrm{~h}$ of incubation. The culture were then mixed gently and incubated for $1 \mathrm{~h}$, harvesting of cells suspension was done follow the standard protocols.

Conventional staining was done using $10 \%$ Giemsa's solution for $10 \mathrm{~min}$. GTG-banding was performed us-
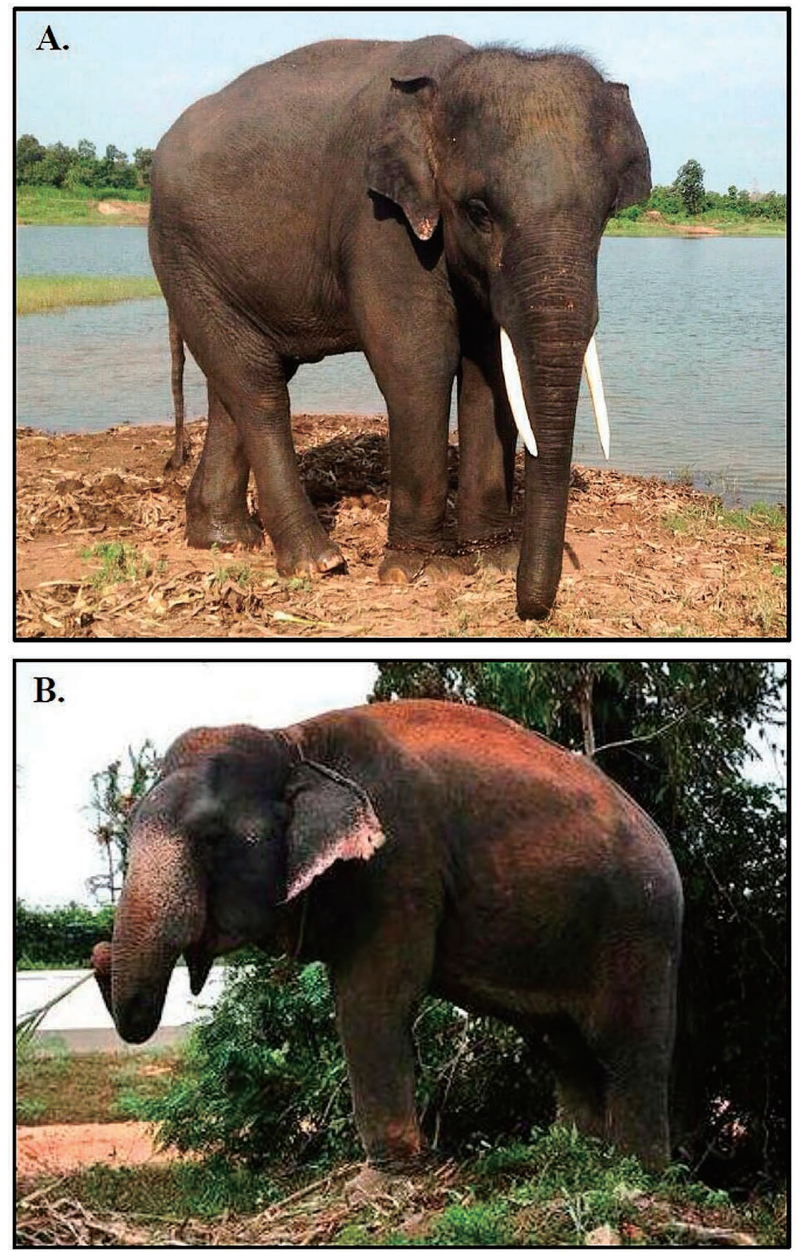

Fig. 1. General characteristics of tusker (A) and tuskless (B) male Asian elephant, Elephas maximus (Proboscidea, Elephantudae).

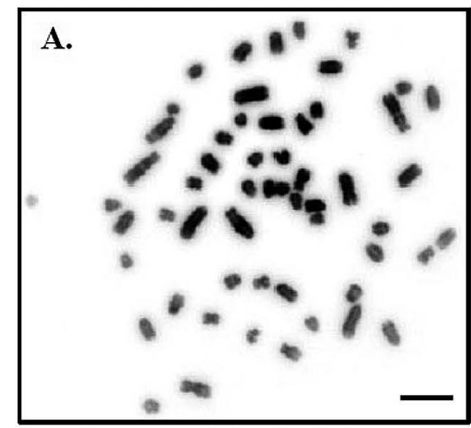

\begin{tabular}{|c|c|c|c|c|c|c|}
\hline & 11 & 18 & 8 & $\mathbf{m a}$ & $=x$ & $\bullet$ \\
\hline & 2 & 3 & 4 & 5 & 6 & 7 \\
\hline & 10 & 18 & 18 & 10 & 10 & 18 \\
\hline & & 10 & 11 & 12 & 13 & 14 \\
\hline 01 & 11 & 12 & $\Delta$ & 10 & $\bullet$ & $\bullet$ \\
\hline 15 & 16 & 17 & 18 & 19 & 20 & 21 \\
\hline 0 & $\Delta$ & $\bullet$ & AQ & $\infty$ & $\bullet$ & 8. \\
\hline & 23 & 24 & 25 & 26 & 27 & $X Y$ \\
\hline & (3) & $\Delta$ & 28 & (1) & 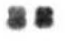 & $\Delta$ \\
\hline & 2 & 3 & 4 & 5 & 6 & 7 \\
\hline & 80 & $\theta 0$ & 80 & a & $\theta$ & $\theta$ \\
\hline$\gamma$ & 9 & 10 & 11 & 12 & 13 & 14 \\
\hline 돕 & $\bullet$ & $\bullet$ & A & 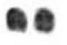 & $\bullet$ & $\bullet$ \\
\hline 15 & 16 & 17 & 18 & 19 & 20 & 21 \\
\hline$\bullet$ & 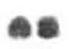 & $\bullet$ & 由 & $\Delta 6$ & at & है. \\
\hline 22 & 23 & 24 & 25 & 26 & 27 & $X Y$ \\
\hline
\end{tabular}

Fig. 2. Metaphase chromosome plates and karyotypes of tusker (A) and tuskless (B) male Asian elephant (Elephas maximus), $2 n=56$ by conventional straining technique. Scale bar $=5 \mu \mathrm{m}$. 
ing $0.025 \%$ trypsin and stained with $10 \%$ Giemsa's. Ag-NOR banding was applied (Howell and Black 1980) using two drops of $2 \%$ gelatin on slides then followed four drops of $50 \%$ silver nitrate, then cover with cover slip and incubated at $60^{\circ} \mathrm{C}$ for $5 \mathrm{~min}$ or until the slide changed to brownish. After that the slides were dipped in distilled water to remove the cover slip and air-dried.

Twenty of clearly metaphase chromosome plates of two male types were photographed. The length of the short arm chromosome (Ls) and the long arm chromosome (Ll) were measured, then the length of the total arm chromosome (LT, LT=Ls+Ll) was calculated. The relative length (RL), centromeric index (CI), standard deviation (SD) of RL and CI were computed (Chaiyasut 1989). The CI (q/p+q) between $0.50-0.59,0.60-0.69$, $0.70-0.89$, and $0.90-0.99$ were described as metacentric, submetacentric, acrocentric and telocentric chromosomes, respectively.

Results and discussion

The diploid chromosome number of both tusker and tuskless male Asian elephant in the present study was 56 (Fig. 2A and 2B), which is in accordance with both L. africana and E. maximus in the previous reports (Hungerford et al. 1966, Norberg 1969, Hösli and Thürig

Table 1. Cytogenetic reviews of the Asian elephant (Elephas maximus).

\begin{tabular}{|c|c|c|c|c|c|}
\hline \multirow{2}{*}{ Title } & \multicolumn{4}{|c|}{ Technique } & \multirow{2}{*}{ Reference } \\
\hline & Con. & G-band & C-band & FISH & \\
\hline $\begin{array}{l}\text { Chromosomes of three elephants, two Asian (Elephus maximus) and one } \\
\text { African (Loxodonta africana) }\end{array}$ & / & - & - & - & Hungerford et al. 1966 \\
\hline $\begin{array}{l}\text { The chromosome of the Indian female elephant (Elephus indiaus syn. } \\
\text { E. maximus) }\end{array}$ & / & - & - & - & Norberg 1969 \\
\hline Elephus maximus (Indian or Asian elephant) $2 n=56$ & 1 & - & - & - & Hsu and Benirschke 1971a \\
\hline Chromosome profile of Indian elephants (Elephus maximus indicus) & / & - & - & - & Sakthikumar et al. 1990 \\
\hline $\begin{array}{l}\text { Electrophoretic and chromosomal variation in captive Asian elephants } \\
\text { (Elephus maximus) }\end{array}$ & - & - & / & / & Hartl et al. 1995 \\
\hline $\begin{array}{l}\text { The GTG-banding karyotype and telomere FISH in Asian elephant (Elephus } \\
\text { maximus) }\end{array}$ & / & / & - & / & Suwattana et al. 2000 \\
\hline $\begin{array}{l}\text { Comparative cytogenetics of the African elephant (Loxodonta africana) and } \\
\text { Asiatic elephant (Elephus maximus) }\end{array}$ & - & / & / & - & Houck et al. 2001 \\
\hline $\begin{array}{l}\text { Karyotype evolution in mammals: A reappraisal by comparative chromo- } \\
\text { some painting }\end{array}$ & - & - & - & / & Graphodatsky et al. 2002 \\
\hline $\begin{array}{l}\text { Cytogenetic and random amplified polymorphism DNA analysis of subspe- } \\
\text { cies of Asian elephants (Elephus maximus) }\end{array}$ & - & / & / & / & Sharudin 2005 \\
\hline
\end{tabular}

Remarks: Con. $=$ conventional staining and - not available.

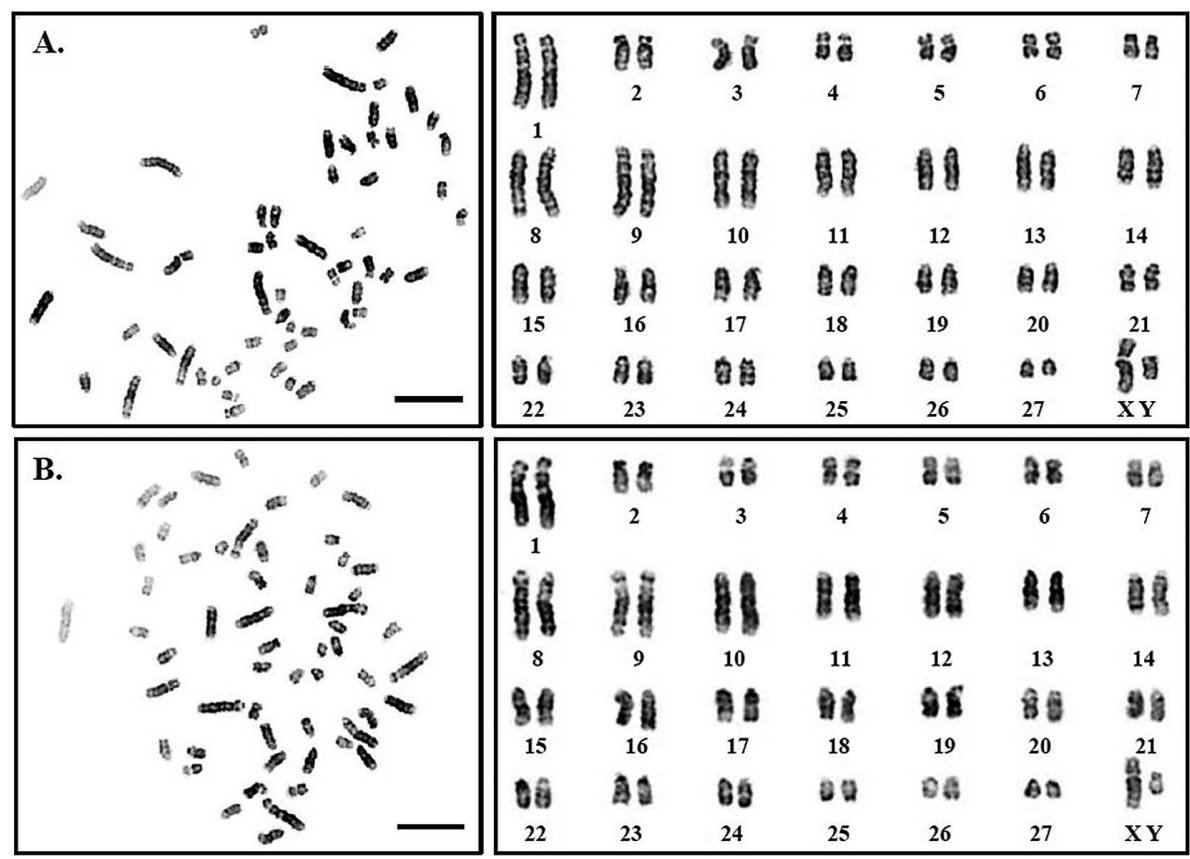

Fig. 3. Metaphase chromosome plates and karyotypes of tusker (A) and tuskless (B) male Asian elephant (Elephas maximus), $2 n=56$ by GTG-banding technique. Scale bar $=5 \mu \mathrm{m}$. 

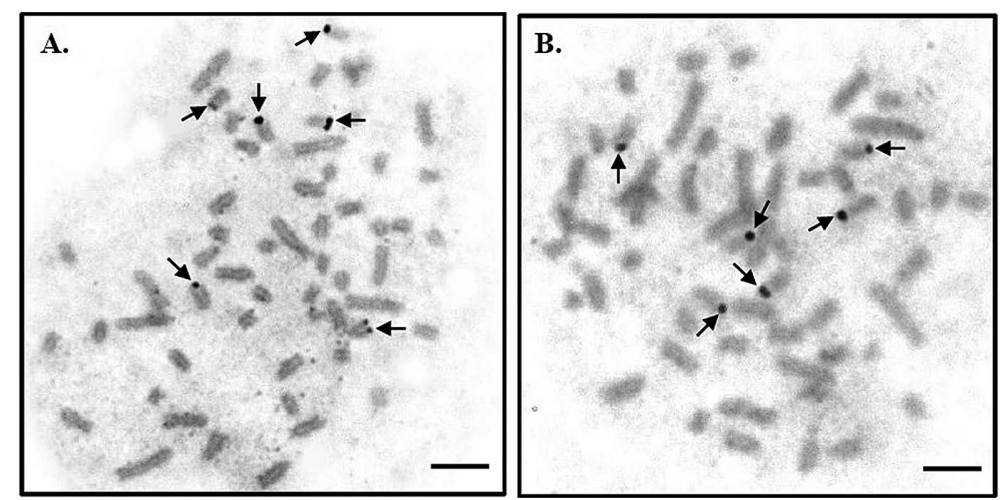

Fig. 4. Metaphase chromosome plates of tusker (A) and tuskless (B) male Asian elephant (Elephas maximus), $2 n=56$ by $\mathrm{Ag}$ NOR banding technique. Arrows indicate nucleolar organizer regions/NORs (Scale bar $=5 \mu \mathrm{m}$ ).

1970, Thürig 1970, Hsu and Benirschke 1971a, 1971b, Wallace 1978, Sakthikumar et al. 1990, Hartl et al. 1995, Suwattana et al. 2000, Houck et al. 2001, Graphodatsky et al. 2002, Sharudin 2005) (Table 1). The fundamental numbers (NF) was 72 in both tusker and tuskless male Asian elephant. It is difference from the report of Sharudin (2005) which demonstrated that NF of Asian elephant is as 66 in both male and female.

The standardize karyotype of both elephants were consisted of one pair of large acrocentric, four pairs of large telocentric, one pair of medium submetacentric, one pair of medium acrocentric, six pairs of medium telocentric, two pairs of small metacentric, two pairs of small submetacentric, and 10 pairs of small telocentric homologous chromosomes. The autosome set was divided into two groups: one contained bi-armed chromosomes (seven chromosome pairs) and the other carried mono-armed chromosomes (20 chromosome pairs). It is not consistent to the report of Suwattana et al. (2000) which revealed that Asian elephant has autosomes as following; six pairs of bi-armed chromosomes and the rest of 21 chromosome pairs were telocentric. Moreover, Sharudin (2005) demonstrated that Asian elephant has six pairs of submetacentric, 10 pairs of large acrocentric, and the remaining 11 pairs are characterized as small acrocentric chromosomes.

The $\mathrm{X}$ sex-chromosome was a medium metacentric and the $\mathrm{Y}$ sex-chromosome was a small acrocentric chromosome. The result is difference from the report of Suwattana et al. (2000) which demonstrated that the medium submetacentric X chromosome and small telocentric Y chromosome of Asian elephant. Furthermore, Sharudin (2005) also reported that the sex-chromosomes of Asian elephant had the largest submetacentric X chromosome while the $\mathrm{Y}$ chromosome is a small acrocentric.

On the basis of classical cytogenetic studies herein by conventional, GTG-banding and Ag-NORs banding techniques could not be distinguished the karyotype of tusker and tuskless male Asian elephants. The GTG banded idiogram representing of both male elephants was shown in Fig. 5. GTG-banding revealed that number

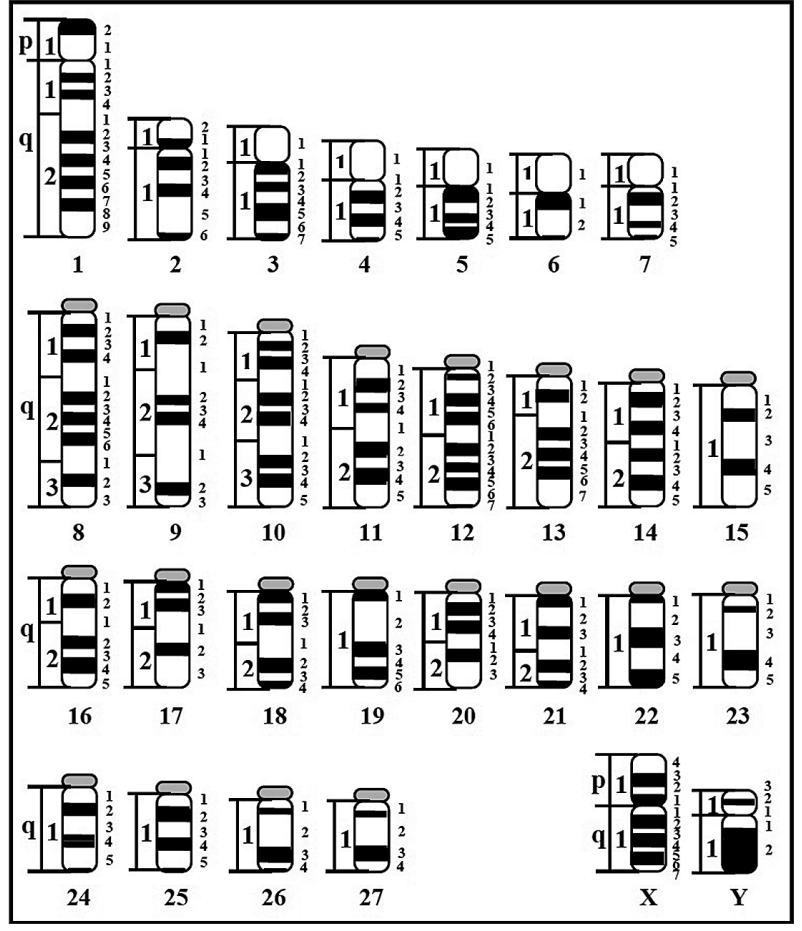

Fig. 5. Idiogram showing lengths and shapes of chromosomes of Asian elephant (Elephas maximus), $2 n=56$ by GTG-banding technique.

of GTG-bands on 1 set of haploid chromosomes, which includes autosomes, $\mathrm{X}$ and $\mathrm{Y}$ chromosomes, is 225 bands. (Fig. 3A and 3B). GTG-banding technique provide a clearly chromosome band which represent in dark band (heterochromatin) and light band (euchromatin) regions on chromosome. Thus, the haploid set of banded Asian elephant consist of 27 autosomes include $\mathrm{X}$ and $\mathrm{Y}$ chromosomes. However, some chromosomes show not clearly identify because of some band has variation. As above the chromosome band scoring is represent by approximate band that appear. However, the GTG-banded idiogram of Asian elephant (Suwattana et al. 2000) and particularly, the idiograms of reciprocal chromosome painting among human and African elephant have already been reported (Frönicke et al. 2003, Yang et al. 
Table 2. Mean length of short arm chromosomes (Ls), long arm chromosomes (L1), total arm chromosomes (LT), relative length (RL), centromeric index (CI), and standard deviation of RL, CI from 20 metaphases of tusker and tuskless male Asian elephant (Elephas maximus), $2 n=56$.

\begin{tabular}{|c|c|c|c|c|c|c|c|}
\hline Chro. pair & Ls & $\mathrm{Ll}$ & LT & RL & $\mathrm{CI}$ & Chro. size & Chro. type \\
\hline 1 & 0.473 & 2.033 & 2.507 & $0.066 \pm 0.005$ & $0.808 \pm 0.022$ & Large & Acrocentric \\
\hline 2 & 0.345 & 0.959 & 1.305 & $0.035 \pm 0.001$ & $0.734 \pm 0.012$ & Medium & Acrocentric \\
\hline 3 & 0.409 & 0.916 & 1.325 & $0.035 \pm 0.002$ & $0.693 \pm 0.034$ & Medium & Submetacentric \\
\hline 4 & 0.449 & 0.702 & 1.151 & $0.031 \pm 0.002$ & $0.610 \pm 0.025$ & Small & Submetacentric \\
\hline 5 & 0.420 & 0.599 & 1.019 & $0.027 \pm 0.002$ & $0.589 \pm 0.047$ & Small & Metacentric \\
\hline 6 & 0.444 & 0.529 & 0.972 & $0.026 \pm 0.003$ & $0.546 \pm 0.033$ & Small & Metacentric \\
\hline 7 & 0.366 & 0.601 & 0.967 & $0.026 \pm 0.003$ & $0.623 \pm 0.027$ & Small & Submetacentric \\
\hline 8 & 0.000 & 2.248 & 2.248 & $0.060 \pm 0.005$ & $1.000 \pm 0.000$ & Large & Telocentric \\
\hline 9 & 0.000 & 2.292 & 2.292 & $0.059 \pm 0.004$ & $1.000 \pm 0.000$ & Large & Telocentric \\
\hline 10 & 0.000 & 2.030 & 2.030 & $0.054 \pm 0.002$ & $1.000 \pm 0.000$ & Large & Telocentric \\
\hline 11 & 0.000 & 1.720 & 1.720 & $0.045 \pm 0.002$ & $1.000 \pm 0.000$ & Large & Telocentric \\
\hline 12 & 0.000 & 1.522 & 1.522 & $0.040 \pm 0.001$ & $1.000 \pm 0.000$ & Medium & Telocentric \\
\hline 13 & 0.000 & 1.516 & 1.516 & $0.040 \pm 0.002$ & $1.000 \pm 0.000$ & Medium & Telocentric \\
\hline 14 & 0.000 & 1.373 & 1.373 & $0.037 \pm 0.002$ & $1.000 \pm 0.000$ & Medium & Telocentric \\
\hline 15 & 0.000 & 1.396 & 1.396 & $0.036 \pm 0.001$ & $1.000 \pm 0.000$ & Medium & Telocentric \\
\hline 16 & 0.000 & 1.265 & 1.265 & $0.034 \pm 0.001$ & $1.000 \pm 0.000$ & Medium & Telocentric \\
\hline 17 & 0.000 & 1.272 & 1.272 & $0.034 \pm 0.001$ & $1.000 \pm 0.000$ & Medium & Telocentric \\
\hline 18 & 0.000 & 1.116 & 1.116 & $0.030 \pm 0.001$ & $1.000 \pm 0.000$ & Small & Telocentric \\
\hline 19 & 0.000 & 1.085 & 1.085 & $0.029 \pm 0.000$ & $1.000 \pm 0.000$ & Small & Telocentric \\
\hline 20 & 0.000 & 1.069 & 1.069 & $0.028 \pm 0.001$ & $1.000 \pm 0.000$ & Small & Telocentric \\
\hline 21 & 0.000 & 1.066 & 1.066 & $0.028 \pm 0.003$ & $1.000 \pm 0.000$ & Small & Telocentric \\
\hline 22 & 0.000 & 1.064 & 1.064 & $0.028 \pm 0.001$ & $1.000 \pm 0.000$ & Small & Telocentric \\
\hline 23 & 0.000 & 1.070 & 1.070 & $0.028 \pm 0.001$ & $1.000 \pm 0.000$ & Small & Telocentric \\
\hline 24 & 0.000 & 0.973 & 0.973 & $0.026 \pm 0.002$ & $1.000 \pm 0.000$ & Small & Telocentric \\
\hline 25 & 0.000 & 0.894 & 0.894 & $0.024 \pm 0.001$ & $1.000 \pm 0.000$ & Small & Telocentric \\
\hline 26 & 0.000 & 0.834 & 0.834 & $0.022 \pm 0.001$ & $1.000 \pm 0.000$ & Small & Telocentric \\
\hline 27 & 0.000 & 0.810 & 0.810 & $0.020 \pm 0.001$ & $1.000 \pm 0.000$ & Small & Telocentric \\
\hline $\mathrm{X}$ & 0.722 & 0.753 & 1.474 & $0.039 \pm 0.004$ & $0.512 \pm 0.031$ & Medium & Metacentric \\
\hline $\mathrm{Y}$ & 0.288 & 0.888 & 1.176 & $0.031 \pm 0.001$ & $0.752 \pm 0.041$ & Small & Acrocentric \\
\hline
\end{tabular}

Remark: Chro. $=$ chromosome.

2003), here we presented once more which allowed us easily pairing of the homologous chromosomes and will be useful for further gene mapping and molecular cytogenetic studies in Asian elephant.

The objective of Ag-NOR banding technique is to reach out the nucleolar organizer regions/NORs which is the representative location of genes (loci) that function in ribosome synthesis (18S and $28 \mathrm{~S}$ ribosomal RNA) (Sharma et al. 2002). If these regions were active during the interphase prior to mitosis, they can be detected by silver nitrate staining (Howell and Black 1980) since it specifically stains a set of acidic protein related to ribosomal synthesis process; this technique actually reveals active NORs (Ag-NORs) and not the rDNA associated to NORs (Jordan 1987). Last but not least, our present study was the first time that reported of the NORs bearing chromosome which has been characterized for tusker and tuskless male Asian elephants that found on one pair of small submetacentric and two pairs of small telocentric chromosomes (Fig. 4A and 4B). It is difference to the publish of Sharudin (2005) that showed Ag-NOR banding revealed active nucleolar organizing regions chromosomes pairs $2,4,13$, and 17 (four chromosome pairs).

The standardize karyotype, idiogram and the basic cytogenetic data obtained should serve as a basis for future chromosome analyses of Asian elephant populations and for comparative cytogenetic studies with other relate species. However, our results found that both of elephants karyotype were slightly difference from those previous reports according to the parameters (Ls, Ll, LT, RL, and CI) from 20 well spread metaphase chromosome plates (Table 2). The karyotype formula could be deduced as $2 n$ (diploid) $=\mathrm{L}_{2}^{\mathrm{a}}+\mathrm{L}_{8}^{\mathrm{t}}+\mathrm{M}_{2}^{\mathrm{sm}}+\mathrm{M}_{2}^{\mathrm{a}}+\mathrm{M}_{12}^{\mathrm{t}}+\mathrm{S}_{4}^{\mathrm{m}}+$ $\mathrm{S}_{4}^{\mathrm{sm}}+\mathrm{S}_{20}^{\mathrm{t}}+\mathrm{XY}(4 \mathrm{~m}+6 \mathrm{sm}+4 \mathrm{a}+40 \mathrm{t}+\mathrm{XY})$.

\section{Acknowledgements}

The authors are grateful to thank Toxic Substances in Livestock and Aquatic Animals Research Group, Khon Kaen University and Rajamangala University of Technology Isan, Surin Campus for the financial support.

\section{References}

Chaiyasut, K. 1989. Cytogenetics and Cytotaxonomy of the Family Zephyranthes. Department of Botany, Faculty of Science, Chulalongkorn University, Bangkok.

Frönicke, L., Wienberg, J., Stone, G., Adams, L. and Stanyon, R. 2003. Towards the delineation of the ancestral eutherian genome organization: Comparative genome maps of human and the African elephant (Loxodonta africana) generated by chromosome 
painting. Proc. Biol. Sci. 270: 1331-1340.

Graphodatsky, A., Perelman, P., Alkalaeva, E., Trifonov, V., Shtukkert, A., Serdukova, N., Biltueva, L., Vorobieva, N., FergusonSmith, M. A., Yang, F., O’Brien, P., Robinson, T. J., Suwattana, D. and Sharipov, I. K. 2002. Karyotype evolution in mammals: A reappraisal by comparative chromosome painting. In: Proceedings of the Third International Conference on Bioinformatics of Genome Regulation and Structure (BGRS'2002), Novosibirsk.

Hartl, G. B., Kurt, F., Hemmer, W. and Nadlinger, K. 1995. Electrophoretic and chromosomal variation in captive Asian elephants (Elephas maximus). Zoo Biol. 14: 87-95.

Hösli, P. and Thürig, D. 1970. The karyotype of the male African elephant: Loxodonta africana. Mammal Chromo. News 11: 110-112.

Houck, M. L., Kumamoto, A. T., Gallagher, D. S. Jr. and Benirschke, K. 2001. Comparative cytogenetics of the African elephant (Loxodonta africana) and Asiatic elephant (Elephas maximus). Cytogenet. Cell Genet. 93: 249-252.

Howell, W. M. and Black, D. A. 1980. Controlled silver-staining of nucleolus organizer regions with a protective colloidal developer: A 1-step method. Experientia 36: 1014-1015.

Hsu, T. C. and Benirschke, K. 1971a. Elephas maximus (Indian or Asian elephant). In: Hsu, T. C. and Benirschke, K. (eds.). An Atlas of Mammalian Chromosomes, Vol. 5. Springer-Verlag, New York. pp. 153-155.

Hsu, T. C. and Benirschke, K. 1971b. Loxodonta africana (African elephant). In: Hsu, T. C. and Benirschke, K. (eds.). An Atlas of Mammalian Chromosomes, Vol. 6. Springer-Verlag, New York. pp. 177-179.

Hungerford, D. A., Chandra, H. S., Snyder, R. L. and Ulmer, F. A. Jr. 1966. Chromosomes of three elephants, two Asian (Elephas maximus) and one African (Loxodonta africana). Cytogenetics 5: 243-246.

Jordan, G. 1987. At the heart of the nucleolus. Nature 329: 489-490.
Norberg, H. S. 1969. The chromosomes of the Indian female elephant (Elephas indicus syn. E. maximus L.). Hereditas 63: 279-281.

Nowak, R. M. 1999. Walker's Mammals of the World. 6th Ed. John's Hopkins University Press, Baltimore.

Rooney, D. E. 2001. Human Cytogenetics: Constitutional Analysis. Oxford University Press, Oxford.

Sakthikumar, A., Mukundana, G. and Raghunandana, K. V. 1990. Chromosome profile of Indian elephants (Elephas maximus indicus). Indian J. Anim. Sci. 60: 175-182.

Sharma, O. P., Tripathi, N. K. and Sharma, K. K. 2002. A review of chromosome banding in fishes. In: Sobti, R. C. and Obe, G. (eds.). Some Aspects of Chromosome Structure and Functions. Narosa Publishing House, New Delhi. pp. 109-122.

Sharudin, S. B. 2005. Cytogenetic and random amplified polymorphism DNA analysis of subspecies of Asian elephants (Elephas maximus). Master's Thesis, Universiti Putra, Malaysia.

Suwattana, D., Koykul, W., Mahasawangkul, S., Kanchanapangka, S., Joerg, H. and Stranzinger, G. 2000. The GTG-banded karyotype and telomere FISH in Asian elephants (Elephas maximus). Vet. Med. 45: 285-289.

Thürig, D. 1970. Zum Chromosomenbild des Afrikanischen Elefanten (Loxodonta africana) unter Berücksichtigung des Sexchromatins. Acta Anatomica 75: 6-12.

Vaughn, T. A. 1986. Mammalogy, 3rd Ed. W. B. Saunders, Philadelphia.

Wallace, C. 1978. Chromosome analysis in the Kruger National Park: Mitotic and meiotic studies in the African elephant Loxodonta africana. Koedoe 21: 197-200.

Yang, F., Alkalaeva, E. Z., Perelman, P. L., Pardini, A. T., Harrison, W. R., O’Brien, P. C. M., Fu, B., Graphodatsky, A. S., FergusonSmith, M. A. and Robinson, T. J. 2003. Reciprocal chromosome painting among human, aardvark, and elephant (superorder Afrotheria) reveals the likely eutherian ancestral karyotype. Proc. Natl. Acad. Sci. U.S.A. 100: 1062-1066. 